\title{
Spin physics at COSY and beyond
}

\author{
Alexander Nass* on behalf of the ANKE [1], PAX [2] and JEDI [3] collaborations \\ Institut für Kernphysik and JARA-FAME, Forschungszentrum Jülich, 52425 Jülich, Germany \\ E-mail: a.nass@fz-juelich.de
}

\begin{abstract}
Hadron physics aims at a fundamental understanding of all particles and their interactions that are subject to the strong force. Experiments using hadronic probes bear the potential to shed light on open questions that address the structure of hadrons and their interaction, as well as the symmetries of nature. The COoler SYnchrotron COSY at the Forschungszentrum Jülich accelerates protons and deuterons with momenta up to $3.7 \mathrm{GeV} / \mathrm{c}$. In combination with internal polarized Hydrogen and Deuterium targets, the availability of electron and stochastically cooled polarized proton and deuteron beams allows for precision measurements.

This presentation highlights selected recent results from the ongoing spin physics programs at the COSY facility. Spin physics projects reaching into the future, such as the quest for polarized antiprotons, the search for permanent electric dipole moments of protons and deuterons, and a test of time reversal invariance using COSY, are presented as well.
\end{abstract}

X Latin American Symposium on Nuclear Physics and Applications (X LASNPA),

1-6 December 2013

Montevideo, Uruguay

\footnotetext{
* Speaker.
} 


\section{Introduction}

The COSY facility at Forschungszentrum Jülich (Germany) comprises sources for unpolarized and polarized beams, an injector cyclotron (JULIC) and the storage and cooler ring with a circumference of about $184 \mathrm{~m}$ [4]. It stores, accelerates and cools beams of protons and deuterons, which may be polarized, and provides them at internal target stations or extracts them for use at external targets and detectors. With a maximum beam momentum of $3.7 \mathrm{GeV} / \mathrm{c}$, it is well suited for a wide range of hadron physics experiments with hadronic probes - in fact it can be considered the hadron spin physics machine because of its possibilities to produce, accelerate, manipulate and use polarized beams and targets. In the following, three examples are given of the current and future program at COSY.

\section{The mass of the $\eta$-meson}

A new measurement of the mass of the $\eta$-meson was motivated by differing results in measurements using hadronic production compared to measurements using photoproduction or decay products. To achieve a high precision measurement of $m_{\eta}$ from $d p \rightarrow{ }^{3} \mathrm{He} \eta$ it is necessary to determine the beam momentum $p_{\mathrm{d}}$ and the final state momentum $p_{\mathrm{f}}$ precisely [5]. The first task has been accomplished by making use of a depolarizing resonance. The spin of a polarized particle in a storage ring precesses around the normal to the plane of the machine. A horizontal rf-field from a solenoid can induce depolarizing resonances. The depolarizing resonance frequency $f_{\mathrm{r}}$ depends on the revolution frequency of the machine and the kinematical factor $\gamma=E / m c^{2}$ where $E$ is the total energy and $m$ is the mass of the particle. For a planar accelerator,

$$
f_{\mathrm{r}} / f_{0}=\gamma G \pm k
$$

where $G$ is the particles gyromagnetic anomaly $\left(G_{\mathrm{d}}=-0.1429872725 \pm 0.0000000073\right)$ and $k$ is an integer. The combination of the measurements of the revolution frequency $f_{0}=1403832 \pm 6 \mathrm{~Hz}$ (Fig. 1 left) and depolarizing frequency $f_{\mathrm{r}}=1011810 \pm 15 \mathrm{~Hz}$ (Fig. 1 right) allows the evaluation of $\gamma=1.9530 \pm 0.0001$ and hence the beam momentum $p_{\mathrm{d}}=3146.41 \pm 0.17 \mathrm{MeV} / \mathrm{c}$. By exploiting its full geometric acceptance near threshold, it was possible to calibrate the ANKE spectrometer very
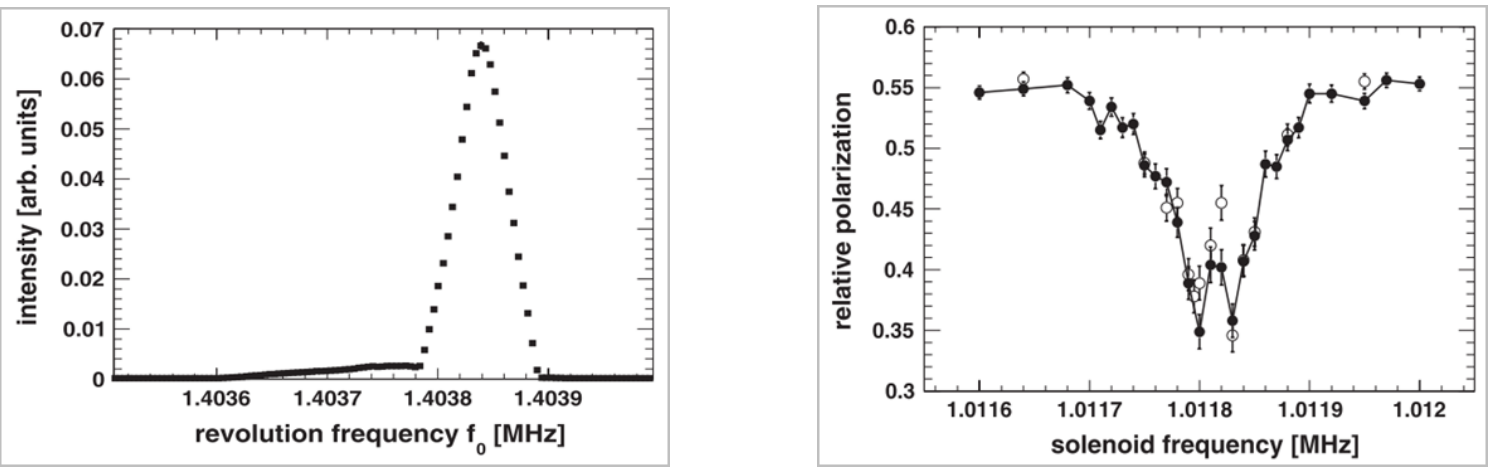

Figure 1: Determination of the revolution frequency (left) and depolarizing resonance frequency (right) (Figures taken from ref. [5]). 
precisely and thus determine the final state momentum $p_{\mathrm{f}}$. With that the mass of the $\eta$ meson was determined to be $m_{\eta}=\left(547.873 \pm 0.005_{\text {stat }} \pm 0.023_{\text {syst }}\right) \mathrm{MeV} / \mathrm{c}^{2}$ which is in excellent agreement with measurements using photoproduction or decay products $[6,7]$.

\section{The quest for polarized antiprotons}

The physics potential for QCD experiments with high-energy polarized antiprotons is enormous but until now many experiments have been impossible because of the lack of a high-luminosity beam. This situation could change with the advent of a stored beam of polarized antiprotons and the realization of a double-polarized, high-luminosity antiproton-proton collider. The collaboration for Polarized Antiproton Experiments (PAX) has formulated the physics program that would be possible with such a facility [8].

In a dedicated experiment at COSY it was shown that spin flip is not a viable tool in polarizing a stored beam [9]. Therefore the experimental approach adopted by the PAX collaboration to produce a beam of polarized antiprotons is based on spin filtering [10], a technique that exploits the spin dependence of the strong interaction. The total cross-section depends on the relative orientation of the spins of the colliding particles, i.e., $\sigma(\uparrow \uparrow) \neq \sigma(\uparrow \downarrow)$. In contrast to the proton-proton system, the experimental basis for predicting the build-up of polarization in a stored antiproton beam by spin filtering is practically non-existent. It is therefore a high priority to perform a series of dedicated spin-filtering experiments using stored antiprotons together with a polarized target, which the PAX collaboration is aiming to undertake at the AD ring at CERN [11].

A spin-filtering measurement with protons using a transversely polarized internal hydrogen target, consisting of an atomic beam source, a storage cell, and a Breit-Rabi type target polarimeter, has been successfully operated in a dedicated low- $\beta$ strait section at COSY. The measurement of the polarization of the circulating proton beam was determined from elastic collision off an unpolarized deuteron cluster target and a pair of silicon tracking telescopes for detection in the ANKE target chamber opposite of the PAX target interaction point (Fig. 2).

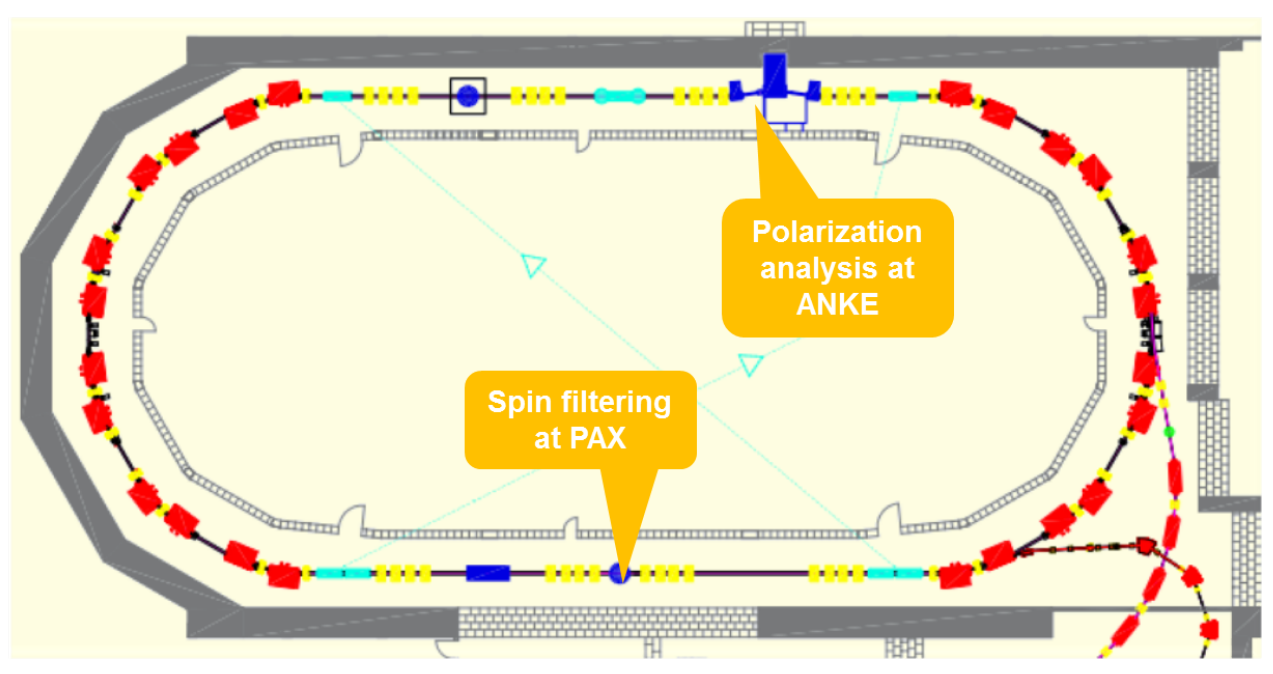

Figure 2: Overview over COSY with the PAX interaction and measurement points. 

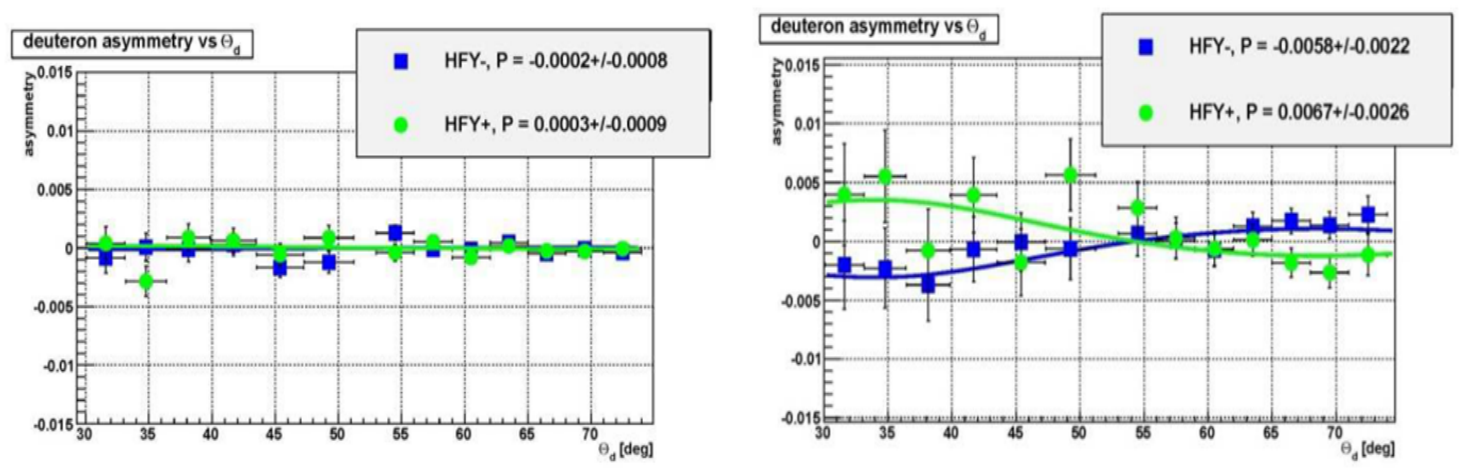

Figure 3: Measured deuteron asymmeties via deflection angle for spin-filter times of $150 \mathrm{~s}$ (left) and $12000 \mathrm{~s}$ (right) (Figures taken from [12]).

The measured asymmetries are shown in Fig. 3. It can be seen that for an unpolarized beam the asymmeties are compatible with zero as expected. This is a good test of the systematics of the experiment. For long spin-filter times different asymmetries for the two different directions of the target polarization are clearly visible (see right panel of Fig. 3).

The resulting polarization of the COSY proton beam is shown in Fig. 4 left [12]. The energy of $49.3 \mathrm{MeV}$ was choosen because of technical restrictions and the well known analyzing powers. From the polarization values a spin-dependent effective polarization buildup cross section $\tilde{\sigma}_{1}$ can be evaluated, which accounts for the fact that only protons scattered at angles larger than the acceptance angle of the storage ring $\theta_{\text {acc }}=6.15 \pm 0.17 \mathrm{mrad}^{1}$ contribute to the spin-filtering process:

$$
\frac{d P}{d t} \approx \frac{1}{\tau_{1}}=\tilde{\sigma}_{1} Q d_{t} f,
$$

where $Q$ denotes the target gas polarization, $d_{\mathrm{t}}$ the target areal density in atoms $/ \mathrm{cm}^{2}$ and $f$ the particle revolution frequency. Using $Q=0.73 \pm 0.05, d_{\mathrm{t}}=(5.5 \pm 0.2) \cdot 10^{13}$ atoms $/ \mathrm{cm}^{2}$ and $f=$
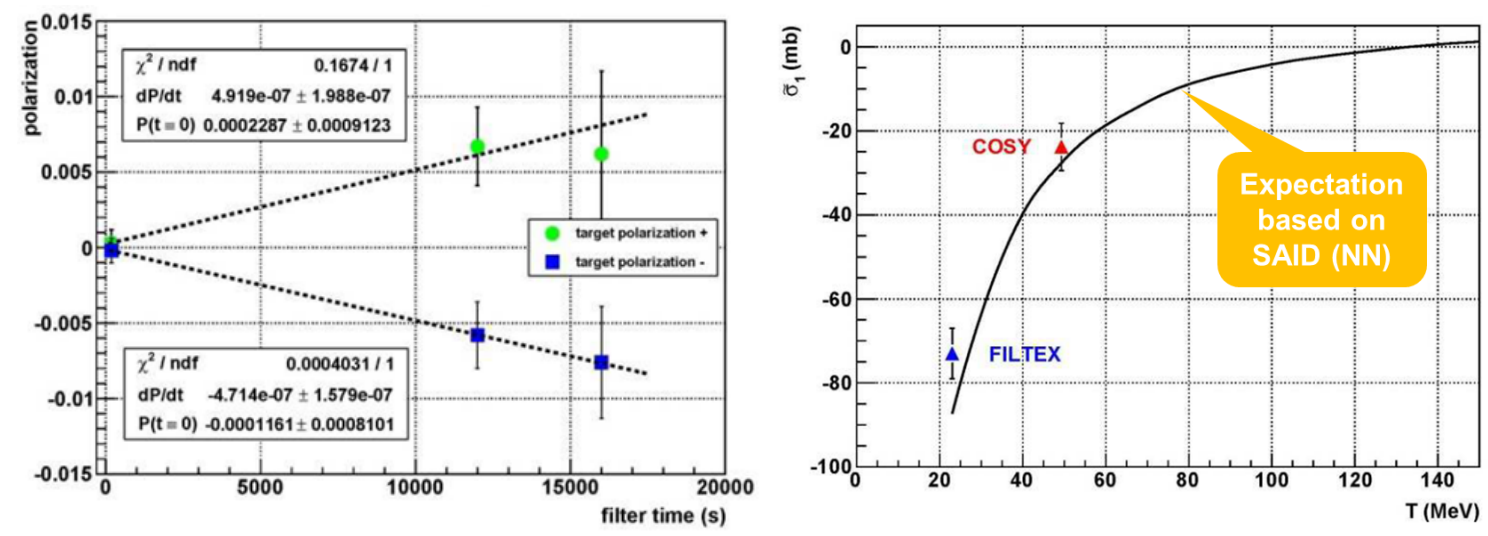

Figure 4: Measured polarization via spin-filter time (left) and the resulting spin-dependent effective polarization buildup cross section $\tilde{\sigma}_{1}$ via experimental energy $T$ (Figures taken from [12]).

\footnotetext{
${ }^{1}$ The storage ring acceptance was measured with a frame system which was moved into the circulating beam [13].
} 
$510032 \mathrm{~Hz}$ the resulting $\tilde{\sigma}_{1}=\left(-23.4 \pm 3.9_{\text {stat }} \pm 1.9_{\text {syst }}\right) \mathrm{mb}$ was measured. This is shown together with the theoretical expectation in Fig. 4 right.

\section{Search for charged particle EDM in storage rings}

Although extremely successful in many aspects, the Standard Model (SM) of particle physics is not capable to explain the apparent matter-antimatter asymmetry of our universe, and thus fails to explain the basis for our existence, for it has way too little $C P$-violation. There are different strategies to hunt for physics beyond the Standard Model. A possible alternative to the high energy frontier exploited at LHC is to employ novel methods which offer very high precision and sensitivity. Permanent electric dipole moments violate both time reversal and parity invariance, and, assuming the validity of $C P T$ invariance, are $C P$-violating. As further explained below searches for permanent electric dipole moments of protons, deuterons and heavier nuclei provide highest sensitivity for the exploration of physics beyond the SM.

It is essential to perform electric dipole moment (EDM) measurements on different targets with similar sensitivity in order to unfold the underlying physics and to explain the baryogenesis. While neutron EDM experiments are pursued at many different locations worldwide (essentially, wherever neutrons are available), no such measurements are conducted yet for proton and other light nuclei. Table 1 shows the current limits and future goal of EDM measurements.

It should be noted that EDM measurements of proton, deuteron, and ${ }^{3} \mathrm{He}$ could be performed in one-and-the same unique machine. The method is based on the spin precession of a particle due to its magnetic and electric dipole moments in external electromagnetic fields, while circulating in a storage ring. Freezing the horizontal spin motion, i.e., forcing the particles' spin to always point along the direction of motion, cancels the $(g-2)$ precession. The build-up of a vertical polarization component $\vec{S}$ in the beam due to:

$$
\frac{\mathrm{d} \vec{S}}{\mathrm{~d} t} \propto \vec{E} \times \vec{d}
$$

indicates the signal for a finite $\operatorname{EDM}(\vec{d})$ of the orbiting particles [14]. Direct measurements of nuclear EDMs of the proton and other charged particles in a dedicated electromagnetic storage ring aiming at a statistical sensitivity of $\sigma_{d} \approx 2.5 \times 10^{-29} \mathrm{ecm}$ per year with the potential of an actual particle-EDM discovery require a special new type of storage ring which incorporates electrostatic

\begin{tabular}{l|l|l|l|l} 
Particle & Current Limit & Goal & $d_{n}$ equiv. & reference \\
\hline Neutron & $<1.6 \times 10^{-26}$ & $\approx 10^{-28}$ & $10^{-28}$ & {$[15]$} \\
${ }^{199} \mathrm{Hg}$ & $<3.1 \times 10^{-29}$ & $10^{-29}$ & $10^{-26}$ & {$[16]$} \\
${ }^{129} \mathrm{Xe}$ & $<6.0 \times 10^{-27}$ & $\approx 10^{-30}-10^{-33}$ & $\approx 10^{-26}-10^{-29}$ & {$[17]$} \\
Proton & $<7.9 \times 10^{-25}$ & $\approx 10^{-29}$ & $10^{-29}$ & {$[16]$} \\
Deuteron & & $\approx 10^{-29}$ & $3 \times 10^{-29}-5 \times 10^{-31}$ &
\end{tabular}

Table 1: Current EDM limits in units of [e.cm], and long-term goals for the neutron, ${ }^{199} \mathrm{Hg},{ }^{129} \mathrm{Xe}$, proton, and deuteron are given here. Neutron equivalent values indicate the EDM value for the neutron to provide the same physics reach as the indicated system. 
deflectors at large electric fields $(E \approx 20 \mathrm{MV} / \mathrm{m})$, which constitutes a major investment. Therefore as a first step a direct measurement of an upper limit of $\sigma_{d} \approx 10^{-24} \mathrm{e} \cdot \mathrm{cm}$ for the proton and deuteron EDM using a normal magnetic storage ring like COSY seems feasible.

COSY has a history of highly successful operation of cooled polarized beams and targets in fact, COSY is a unique facility for spin physics with hadronic probes on a world-wide scale. It is the preferential place where the technology for a dedicated EDM ring can be developed and tested. One important aspect of a successful EDM measurement with a polarized, charged-particle beam is the coherence of the spin motion of the particles. For this reason, a series of dedicated studies is being performed to examine the effects of emittance spread on the spin coherence time. First experiments show that the in-plane (horizontal) polarization of the circulating proton beam can be measured by a novel polarimeter system [18] and that the coherence of the spin precession is sufficiently long (>1000 s) to perform a direct EDM measurement.

\section{Time reversal invariance test at COSY}

Time-reversal symmetry is one of the most fundamental symmetries in nature. $C P$-violation phenomena, which can be regarded as equivalent of $T$-violation on the basis of the $C P T$ theorem, have been observed in $K^{0}$ and $B$ systems. In the standard model (SM) $C P$ violation appears as a complex phase parameter in the Cabibo-Kobayashi-Maskawa (CKM) matrix. Currently all observed CP violating phenomena appear to be consistent with the SM. However, as mentioned before, the standard model $C P$ violation is by orders of magnitude too small to account for the apparent asymmetry between matter and anti-matter in the universe.

It is proposed to use COSY as an accelerator, ideal zero degree spectrometer, and detector. The effects of the breaking of time-reversal invariance can be searched for simultaneously in measurements of total cross sections using a beam-current transformer (BCT), and studies of differential observables using the PAX detection system. A status report about an earlier preparatory experiment carried out at COSY is given in [19]. The test beam time (Oct. 2006) has shown that the accuracy of the standard BCT is not sufficient to reach the necessary precision of $10^{-6}$ in a timereversal non-invariance test. Recently a new BCT has been developed [20] which allows to reach the mentioned accuracy.

\section{Summary}

COSY is the only facility worldwide which provides polarized beams of protons and deuterons in the intermediate energy range for internal and external experiments. Together with existing and new detection systems, it constitutes an indispensable facility for studies of hadron physics with hadronic probes. It constitutes an important test facility for preparatory tests for the upcoming FAIR project at GSI. The search for EDMs of protons, deuterons, and other light nuclei presents both an exceptional opportunity for world-class physics and a tremendous challenge for experiment and theory.

\section{References}

[1] http://collaborations.fz-juelich.de/ikp/anke/ 
[2] http://collaborations.fz-juelich.de/ikp/pax/

[3] http://collaborations.fz-juelich.de/ikp/jedi/

[4] R. Maier, Nuclear Instruments and Methods A390 (1997), 1-8

[5] A. Kacharava and C. Wilkin, Nuclear Physics News, Vol. 23 No. 2 (2013)

[6] P. Goslawski et al., Phys. Rev. ST Accel. Beams 13 (2010) 022803.

[7] P. Goslawski et al., Phys. Rev. D 85 (2012) 112011.

[8] V. Barone et al. (PAX-Collaboration) 2005 (Preprint hep-ex/0505054)

[9] D. Oellers et al., Phys. Lett. B674 (2009) 269-275

[10] V. Dmitriev, A. Milstein and S. Salnikov, Phys.Lett. B690 (2010) 427-430

[11] C. Barschel et al. (PAX-Collaboration) (Preprint 0904.2325)

[12] W. Augustyniak et al., Physics Letters B 718 (2012), 64-69

[13] K. Grigoriev et al., Nucl. Instr. and Methods A 599 (2009) 130-139.

[14] Y. K. Semertzidis (Storage Ring EDM Collaboration), AIP Conf. Proc. 1182 (2009) 730-736

[15] C. Baker et al., Phys. Rev. Lett. 98 (2007) 149102

[16] W. Griffith et al., Phys. Rev. Lett. 102 (2009) 101601

[17] M. A. Rosenberry and T. E. Chupp, Phys. Rev. Lett. 86 (2001) 22-25

[18] P. Lenisa, E. J. Stephenson and G. Guidoboni, PoS (PSTP 2013) 013

[19] D. Eversheim et al., Hyperfine Interact. 193 (2009), 335-339

[20] Bergoz Instrumentation, Integrating Current Transformer, User Manual rev. 3.0 (2010) 